\title{
Next steps for meeting the needs of people with severe mental illness in low- and middle-income countries
}

\author{
C. Hanlon ${ }^{1,2 *}$ \\ ${ }^{1}$ Department of Psychiatry, School of Medicine, College of Health Sciences, Addis Ababa University, PO 9086, Addis Ababa, Ethiopia \\ ${ }^{2}$ Health Services and Population Research Department, Centre for Global Mental Health, Institute of Psychiatry, Psychology and Neuroscience, \\ King's College London, UK
}

\begin{abstract}
The explicit inclusion of mental health within the Sustainable Development Goals is a welcome development, borne out of powerful advocacy using public health, economic and human rights arguments. As funding comes on line for scaleup of evidence-based mental health care by task-sharing with primary care, it is time to take stock about care for people affected by severe mental illness (SMI). The existing evidence base for task shared care for SMI provides an imperative to get started, but is skewed towards relatively more affluent and urban populations in middle-income countries where specialist mental health professionals provide most of the care. Randomised, controlled trials and rigorous implementation research on task shared service models are underway which will go some way to improving understanding of the quality, safety, effectiveness and acceptability of more widely generalisable care for people with SMI. A sub-group of people with SMI have more complex and long-term needs for care, with a high risk of homelessness, imprisonment and human rights violations as family and social supports become overwhelmed. Case studies from non-governmental organisations provide examples of holistic approaches to rehabilitation, recovery and empowerment of people with SMI, but rigorous comparative studies are needed to identify the most efficient, effective and scalable approaches to care. Health system constraints are emerging as the over-riding barriers to successful task-sharing, highlighting a need to develop and evaluate chronic care models for people with SMI that succeed in reducing premature mortality, improving wellbeing and achieving better social outcomes. Addressing these evidence gaps is essential if task-sharing mental health care is going to deliver on its promise of promoting recovery for the full range of people affected by SMI.
\end{abstract}

First published online 20 December 2016

Key words: Severe mental illness, mental disorders, sub-Saharan Africa, community mental health care, task-shifting, primary healthcare.

In low- and middle-income countries (LMICs), the majority of people with severe mental illness (SMI: including schizophrenia and bipolar disorder) do not have access to evidence-based treatment (Wang et al. 2007). Often in the absence of alternatives, many people with SMI seek help from traditional or faith healers (Abbo, 2011). The inadequacy of existing care systems is demonstrated by studies which show that people with SMI continue to experience a high burden of disability (Gureje \& Bamidele, 1999), poverty and food insecurity (Trani et al. 2015), homelessness (Fekadu et al. 2014a), stigma, discrimination and human rights violations (Thara \& Srinivasan, 2000), and poorer physical health and premature mortality (Ran et al. 2007; Fekadu et al. 2014b).

* Address for correspondence: Health Services and Population Research Department, Centre for Global Mental Health, Institute of Psychiatry, Psychology and Neuroscience, King's College London, UK.

(Email: Charlotte.hanlon@kcl.ac.uk)
Improving access to treatment for people with SMI through task-sharing with primary healthcare has been given high priority within the World Health Organisation's mental health Gap Action Programme (mhGAP) (World Health Organization, 2008). Task-sharing is when circumscribed components of care are delegated to existing or new cadres of worker who either have less training or more narrowly focused training than specialist care providers (Hanlon et al. 2016). There is an important distinction between task-'shifting' and task-'sharing'; with task-' sharing' emphasising an ongoing, shared responsibility for care by specialist workers. In mhGAP, primary care and general health care workers receive brief (up to 10 days) of training in evidence-based guidelines for priority mental, neurological and substance use disorders (World Health Organization, 2010). The guidelines for psychosis and bipolar disorder include prescription of psychotropic medication (antipsychotics, mood-stabilisers, antidepressants and anxiolytics), provision of psychosocial care and consultation or referral to specialists when needed. 


\section{Challenges for evaluations of task-sharing in SMI}

Although there is accumulating evidence to support task-sharing approaches to interventions for depression and other common mental disorders (Padmanathan \& De Silva, 2013), there is much less agreement about the nature and extent of task-shared care required for people with SMI (van Ginneken et al. 2013). Historically, initiatives using task-sharing approaches to expand mental health care have been critiqued for lack of systematic evaluation, particularly at the level of the individual patient (Cohen, 2001; Hanlon et al. 2010). The paucity of trials on SMI care in LMICs has been highlighted (Purgato et al. 2012).

There has been relatively less international attention given to developing evidence on optimal care for people with SMI compared with people with depression for various reasons: (1) depression is highly prevalent and thus makes a larger contribution to populationlevel disease burden and lost economic productivity compared with SMI (Chisholm et al. 2016), even though it is often less disabling for the individual, (2) treatment for depression more often restores a person to full functioning whereas a proportion of people with SMI will remain economically unproductive (Strand et al. 2015), (3) global efforts to improve treatment and control of non-communicable diseases and chronic communicable diseases have led to a focus on the detrimental impact of untreated co-morbid depression (Ngo et al. 2013) and relative neglect of the high co-morbidity of chronic disorders in people with SMI and (4) depression carries less stigma than SMI and advocacy messages targeted at donors may be more palatable when linked to depression.

Research into SMI care in LMICs has particular challenges. Usually studies require the involvement of scarce specialist mental health professionals for diagnosis and evaluation of symptom severity, for which self-report or lay-administered measures are inadequate. Particularly in areas of low treatment coverage, participants may lack capacity to consent to involvement in research and ethical review bodies may struggle to understand the necessity of their involvement, with appropriate safeguards, in order to ensure equity ( $\mathrm{Ng}$ et al. 2015). The controversy surrounding the care of people with SMI when they are unable to consent to treatment (Freeman et al. 2015) may be a barrier to research. The strong prioritisation of primary care-based mental health care for research and funding (Collins et al. 2011) has shifted focus away from the role of mental health specialists, even though ongoing specialist input is recognised to be necessary for effective task-sharing in primary care, especially for people with SMI (Thornicroft et al. 2010).

\section{Existing evidence and work-in-progress}

There have been several recent initiatives to evaluate the effectiveness of approaches to task-sharing care for people with SMI in LMICs using randomised controlled trials and rigorous implementation science methodology.

The Programme for Improving Mental health carE (PRIME) is operational in five LMICs (Ethiopia, India, Nepal, South Africa, Uganda) and seeks to evaluate models of mental health care integrated within primary care for psychosis/bipolar disorder, epilepsy, depression and alcohol use disorders (Lund et al. 2012). Extensive formative work and community engagement led to development of mental health care plans, which are now being evaluated at scale (Hanlon et al. 2015). There is a notable difference in the approach to integrated and task-shared care of people with SMI across the PRIME sites. In the best resourced PRIME country, South Africa (an upper middleincome country), specialist mental health care is mostly accessible to the community and the role of primary care has been to receive back-referrals from specialists and to provide a recovery-oriented psychosocial group intervention (Brooke-Sumner et al. 2016). In contrast, in Ethiopia (a low-income country), mental health specialists are scarce and demand for health systembased care is low. Therefore, the Ethiopia mental health care plan focuses on community-based awarenessraising and case detection, with primary care workers (nurses and health officers) tasked with making the diagnosis, initiating treatment (including prescription of psychotropic medication) and providing continuing care, with monthly supervision from a psychiatric nurse (Fekadu et al. 2015a). In India, a mental health specialist is stationed within primary care and provides treatment to people with SMI identified in community outreach by frontline workers (Shidhaye et al. 2015). The cross-country evaluation of the impact of these differently configured task-sharing interventions on clinical, social and economic outcomes in people with SMI will provide valuable information to guide best practice (De Silva et al. 2015).

An alternative, and more conservative, model of facility-based task-shared care for people with SMI is under evaluation in Ethiopia. In the TaSCS trial (Task-Sharing for the Care of Severe mental disorders), people with SMI who have received their initial diagnosis from a mental health professional (usually a psychiatric nurse) have been randomised to be referred back for ongoing care to primary care workers who have received brief training in mhGAP or continue to have hospital-based psychiatric nurse-led out-patient care (Mayston et al. 2015). The TaSCS trial will produce evidence on whether or not this back-referral model of 
task-sharing is non-inferior to specialist led care in terms of clinical and functional outcomes, the costeffectiveness of task-sharing and the quality of care delivered in each setting (Hanlon et al. 2016).

Engagement with traditional and faith healers is often recommended as an essential part of community provision of care for people with SMI, but this is rarely put into practice and even more rarely evaluated (Nortje et al. 2016). In the COllaborative Shared care to IMprove Psychosis Outcome (COSIMPO) trial in Nigeria and Ghana, people with SMI will be allocated randomly to a collaborative care model between alternative care providers (Christian faith healers, Islamic faith healers and traditional healers) and primary health care providers or enhanced care as usual (Gureje, 2016). Impact will measure the experience of abusive practices as well as symptom reduction.

Task-sharing service models with seek to support recovery of people with schizophrenia in the community have been developed. Community-based rehabilitation delivered by lay workers is being evaluated in the RISE trial (Rehabilitation Intervention for people with Schizophrenia in Ethiopia) (Asher et al. 2016). The effect of adjunctive community-based rehabilitation upon functional recovery and social reintegration will be compared with people allocated to nonspecialist, primary care-based mental health care alone. The 'mental health and development' model of task-sharing combines access to a primary care-based psychiatric nurse with community mobilisation, establishment of self-help support groups, livelihood and income-generating opportunities (BasicNeeds, 2009). A pre-post evaluation provided promising evidence of the long-term benefits of this programme upon clinical, social and economic outcomes in people with SMI in rural Kenya (Lund et al. 2013). Comparison with facility-based care alone is now needed to optimise the added value of the development-related activities.

In middle-income countries, task-sharing interventions have typically been linked to specialist mental health care. Involvement of people with SMI as peer support workers working alongside community mental health workers is the model being evaluated in urban centres in Brazil, Chile and Argentina in the RedeAmericas trial (Minoletti et al. 2012). A critical time intervention ('critical time intervention tasksharing') is being delivered to people with SMI at first contact with mental health services to promote a positive engagement with services, integrate people with SMI into primary care as well as specialist care and access community-based social support. In the COmmunity care for People with Schizophrenia in India (COPSI) trial (Chatterjee et al. 2014), a collaborative community-based model of care, comprising psychiatrist-led care augmented by a 12 month personalised, home-based programme delivered by community health workers, was compared with psychiatrist-led care alone. Although overall reductions in disability combined across the three study sites were modest, there was evidence of greater impact in the rural centre. In the STOPS trial in Pakistan (Supervised Treatment in Out-patients for Schizophrenia), out-patient psychiatric care was augmented by family supervision of medication adherence based on the 'DOTS' model used to treat tuberculosis (Farooq et al. 2011). After 12 months, people allocated to STOPS had significantly better adherence as well as clinical and functional outcomes. Task-sharing models of psychoeducation for people with SMI linked to specialist mental health care have also been shown to improve clinical and functional outcomes (Zhao et al. 2015).

\section{What else is needed?}

The trials of task-sharing care for people with SMI, which are underway or recently completed will yield valuable information to guide service designs. However, critical gaps still remain. It is clear that very few evaluations have been, or are being, conducted on fully task-shared care for people with SMI, whereby primary care workers diagnose, initiate treatment and provide ongoing care and specialists provide supervision, consultation and review of complex cases. This is the only type of task-sharing which is likely to increase mental health care coverage in low-income countries and poorly resourced areas of middleincome countries where specialists are scarce. As well as the need to expand the contexts within which such 'full' task-sharing is evaluated (e.g., to more rural settings), there needs to be improved guidance on best emergency care of people with SMI in general healthcare and primary care settings (World Health Organization, 2010). The evidence base in this area from LMICs is almost non-existent (Nadkarni et al. 2015) even though task-shared services will inevitably encounter people presenting in crisis. Bipolar disorder and the management of SMI in perinatal women have also not been the focus of most evaluation studies to date. The relatively greater technical demands of delivering care for people with bipolar disorder in LMICs mean alternative task-sharing approaches are required (Fekadu et al. 2015b).

Health system bottlenecks are increasingly recognised as being critical to the success or otherwise of task-shared care. In a qualitative study with key informants from six LMICs conducted by the Emerald project (Emerging mental health systems in LMICs) (Semrau et al. 2015), system level barriers to successful task-shared care included the lack of a chronic care 
orientation to the health system, weak systems to support primary care workers to deliver mental health care, poor information systems and difficulty tracking people who dropped out of care (Petersen et al. 2016). Previous reviews have highlighted deficiencies in the long-term care of people with SMI in sub-Saharan Africa, with medication doses staying static even when there are changes in clinical state and little focus on recovery and rehabilitation (Hanlon et al. 2010).

The 'chronic care model' is a health system configuration, which has been shown to improve outcomes for chronic disorders in high-income countries (Wagner et al. 2001). This approach has been adapted for LMICs (as the 'innovative care for chronic conditions framework'; ICCCF) (Epping-Jordan et al. 2004) and formative work from Ethiopia indicates that ICCCF has the potential to address many of the system challenges with task-shared care for people with SMI (Mall et al. 2015). The key elements of the ICCCF are as follows: equipping people affected by chronic disorders, their caregivers and communities with the information, access to treatment and support they need in order to maximise their health ('informed, motivated and prepared'); shifting from delivery systems which are geared to respond to acute, infectious illness to those which promote long-term co-ordination and planning of person-centred care ('delivery system design' and information systems); integrating evidence-based guidelines into routine care, supporting access to expertise and orientating health care providers to the changes in clinician-patient relationship needed for long-term care ('organised and well-equipped healthcare teams'); and ensuring conducive policies, legislation and financing to support good leadership and sustainable, quality care.

What might the ICCCF for SMI care look like in a low-income country? At the community level, there would be increased involvement of service users and caregivers in mental health service development and monitoring, models of which are being developed and evaluated (Semrau et al. 2015). Empowerment and engagement of service users can be a powerful vehicle to ensure quality of care, increase awareness and uptake of care, reduce stigma and support selfmanagement in people with SMI. More proactive and flexible approaches to care may require community health workers to deliver medication to homes in the event that the person is too unwell to be conveyed. Mobile phone reminder systems can link facility workers to community workers and co-ordinate home visits to people who have dropped out of care. Facility-based health workers may need to provide outreach for people needing urgent review or to administer depot medication. Evidence-based decision supports need to be extended to include long-term care, including the physical healthcare checks needed for people with SMI and the regular review of functional recovery and setting of rehabilitation goals. There is a potential for electronic systems to track the progress of individuals and support health workers to 'treat to target', i.e., to make care responsive to the changing needs of the person and to ensure that the best possible quality of life is achieved.

Applying the ICCCF to fragmented and weak primary care systems in low-income countries will be challenging and rigorous evaluation is required to ensure that it achieves its goals and does not result in any adverse consequences. There is a need to evaluate quality of care, including the extent to which the person with SMI is involved in decisions pertaining to their care or subject to coercion. ICCCF has great potential to improve physical health care for people with SMI, so physical health outcomes need to be measured, including under-nutrition. The responsiveness of the service to people who drop out of care will also be an important indicator of success, given that adherence to psychotropic medication has been shown to be a key driver of good clinical outcomes (Chatterjee et al. 2014).

Primary care-based treatment cannot meet the needs of all people with SMI, especially those with complex needs, for example, due to treatment-resistant symptoms, challenging behaviours and dependence on others for self-care; the same group who are at highest risk of homelessness, imprisonment, human rights abuses and premature mortality. Specialist-led community mental health care for this sub-group is the norm in high-income countries but, within Africa, has only been tested and evaluated in South Africa (Botha et al. 2014). Examples from the nongovernmental sector indicate that therapeutic centres for people with SMI who are homeless can achieve social re-inclusion, although a small minority continue to require long-term residential care (Eaton et al. 2015). Such centres are run by members of the local community, with strong reliance on volunteers, and combine access to biomedical care, participation in a community, psychoeducation, livelihoods training or involvement in income-generating activities and non-specialist counselling. While continuing to focus on the potential for task-sharing to promote timely treatment and prevent homelessness and social complications of SMI, it is vital that sufficient attention is paid to people with SMI requiring more resource-intensive interventions. A particular challenge is when people with SMI interface with the criminal justice system and may be vulnerable to unfair sentencing and harsh punishments. To counter this there is a need to improve detection of people with SMI in police custody and prisons and ensure 
the adequacy of legal frameworks and procedures to support diversion to mental health care (Almanzar et al. 2015). Other areas requiring focus are access to disability grants and the need for long-term supported living for a sub-group of people with SMI. Given the high costs of these interventions, rigorous evaluation is especially important.

Underpinning efforts to improve the evidence base of care for people with SMI is the need for mental health professionals in LMICs to be trained in a public mental health perspective, with awareness of the need to expand mental health care and interest in identifying contextually appropriate and effective service approaches to achieving this goal. Efforts to build capacity to generate locally applicable evidence have been instrumental in many of the initiatives described in this paper (Lund et al. 2012; Minoletti et al. 2012; Gureje, 2016; Hanlon et al. 2016).

\section{Conclusions}

Task-sharing care for people with SMI holds much potential for expanding coverage of evidence-based care and improving physical health outcomes and social integration. However, in order to achieve the desired goal of universal health care for people with SMI (Patel, 2016), there is a need to develop system interventions to support task-sharing, improve the evidence base in rural and resource-poor LMIC settings and pay greater attention to those people who need more than task-shared care can deliver. Calls have been made for a global fund for people with SMI (Farooq et al. 2016). Only with such commitment of resources and an improved evidence base for care will the needs of people with SMI be met adequately in LMICs.

\section{Acknowledgements}

I acknowledge gratefully the review and comments by Abebaw Fekadu.

\section{Financial support}

I am part-employed on the Programme for Improving Mental health carE (PRIME) Research Programme Consortium funded by UK aid from the UK Government; however, the views expressed do not necessarily reflect the UK Government's official policies.

\section{Conflict of Interest}

None.

\section{References}

Abbo C (2011). Profiles and outcome of traditional healing practices for severe mental illnesses in two districts of Eastern Uganda. Global Health Action 4, 7117. doi: 10.3402/ gha.v3404i3400.7117.

Almanzar S, Katz CL, Harry B (2015). Treatment of mentally Ill offenders in nine developing Latin American Countries. Journal of the American Academy of Psychiatry and the Law 43, 340-349.

Asher L, De Silva M, Hanlon C, Weiss HA, Birhane R, Ejigu DA, Medhin G, Patel V, Fekadu A (2016).

Community-based rehabilitation intervention for people with Schizophrenia in Ethiopia (RISE): study protocol for a cluster randomised controlled trial. Trials 17, 299.

BasicNeeds (2009). Community Mental Health Practice. Seven Essential Features for Scaling up in Low- and Middle-income Countries. BasicNeeds: Bangalore.

Botha UA, Koen L, Galal U, Jordaan E, Niehaus DJ (2014). The rise of assertive community interventions in South Africa: a randomized control trial assessing the impact of a modified assertive intervention on readmission rates; a three year follow-up. BMC Psychiatry 14, 1-10.

Brooke-Sumner C, Lund C, Selohilwe O, Petersen I (2016). Community-based psychosocial rehabilitation for schizophrenia service users in the north west province of South Africa: a formative study. Social Work in Mental Health, 1-35.

Chatterjee S, Naik S, John S, Dabholkar H, Balaji M, Koschorke M, Varghese M, Thara R, Weiss HA, Williams P, McCrone P, Patel V, Thornicroft G (2014).

Effectiveness of a community-based intervention for people with schizophrenia and their caregivers in India (COPSI): a randomised controlled trial. Lancet 383, 1385-1394.

Chisholm D, Sweeny K, Sheehan P, Rasmussen B, Smit F, Cuijpers P, Saxena S (2016). Scaling-up treatment of depression and anxiety: a global return on investment analysis. Lancet Psychiatry 3, 415-424.

Cohen A (2001). The Effectiveness of Mental Health Services in Primary Care: the View from the Developing World. World Health Organization: Geneva.

Collins PY, Patel V, Joestl SS, March D, Insel TR, Daar AS, Bordin IA, Costello EJ, Durkin M, Fairburn C, Glass RI, Hall W, Huang Y, Hyman SE, Jamison K, Kaaya S, Kapur S, Kleinman A, Ogunniyi A, Otero-Ojeda A, Poo M-M, Ravindranath V, Sahakian BJ, Saxena S, Singer PA, Stein DJ, Anderson W, Dhansay MA, Ewart W, Phillips A, Shurin S, Walport M (2011). Grand challenges in global mental health. Nature 475, 27-30.

De Silva M, Rathod S, Hanlon C, Breuer E, Chisholm D, Fekadu A, Jordans M, Petersen I, Prince M, Shidhaye R, Thornicroft G, Kigozi F, Tomlinson M, Lund C, Patel V (2015). Cross country methods to evaluate the coverage and effectiveness of district mental health care plans in 5 low and middle income countries. British Journal of Psychiatry Supplement, S1-S8. doi: 10.1192/bjp.bp.1114.153858.

Eaton J, Des Roches B, Nwaubani K, Winters L (2015). Mental health care for vulnerable people with complex needs in low-income countries: two services in West Africa. Psychiatric Services 66, 1015-1017. 
Epping-Jordan JE, Pruitt SD, Bengoa R, Wagner EH (2004). Improving the quality of health care for chronic conditions. Quality and Safety in Health Care 13, 299-305.

Farooq S, Nazar Z, Irfan M, Akhter J, Gul E, Irfan U, Naeem F (2011). Schizophrenia medication adherence in a resource-poor setting: randomised controlled trial of supervised treatment in out-patients for schizophrenia (STOPS). British Journal of Psychiatry 199, 467-472.

Farooq S, Burns J, Sumathipala A, Naeem F (2016). A global mental health fund for serious mental illness in low-income and middle-income countries. Lancet Psychiatry 3, 495-496.

Fekadu A, Hanlon C, Gebre-Eyesus E, Agedew M, Haddis S, Teferra S, Gebre-Eyesus T, Baheretibeb Y, Medhin G, Shibre T, Workneh A, Tegegn T, Ketema A, Timms P, Thornicroft G, Prince M (2014a). Burden of mental disorders and unmet needs among street homeless people in Addis Ababa, Ethiopia. BMC Medicine 12, 138.

Fekadu A, Medhin G, Kebede D, Alem A, Cleare A, Prince M, Hanlon C, Shibre T (2014b). Excess mortality in severe mental disorders: a 10-year population-based cohort study in rural Ethiopia. British Journal of Psychiatry 206, 289-296.

Fekadu A, Hanlon C, Medhin G, Alem A, Selamu M, Giorgis T, Shibre T, Teferra S, Tegegn T, Breuer E, Patel V, Tomlinson M, Thornicroft G, Prince M, Lund C (2015a). Development of a scalable mental healthcare plan for a rural district in Ethiopia. British Journal of Psychiatry Supplement. doi: 10.1192/bjp.bp.1114.153676.

Fekadu A, Hanlon C, Thornicroft G, Lund C, Kaaya S, Alem A, Gureje O, Cleare AJ, Patel V, Prince MJ (2015b). Care for bipolar disorder in LMICs needs evidence from local settings. Lancet Psychiatry 2, 772-773.

Freeman MC, Kolappa K, Caldas de Almeida JM, Kleinman A, Makhashvili N, Phakathi S, Saraceno B, Thornicroft G (2015). Reversing hard won victories in the name of human rights: a critique of the General Comment on Article 12 of the UN Convention on the Rights of Persons with Disabilities. Lancet Psychiatry 2, 844-850.

Gureje O (2016). COllaborative Shared Care to IMprove Psychosis Outcome (COSIMPO). (ed. clinicaltrials.gov).

Gureje O, Bamidele R (1999). Thirteen-year social outcome among Nigerian outpatient with schizophrenia. Social Psychiatry and Psychiatric Epidemiology 34, 147-151.

Hanlon C, Wondimagegn D, Alem A (2010). Lessons learned in developing community mental health care in Africa. World Psychiatry 9, 185-189.

Hanlon C, Fekadu A, Jordans M, Kigozi F, Petersen I, Shidhaye R, Honikman S, Lund C, Prince M, Raja S, Thornicroft G, Tomlinson M, Patel V (2015). District Mental Health Care Plans for five low- and middle-income countries: commonalities, variations and evidence gaps. British Journal of Psychiatry Supplement 207, s1-s8. doi: 10.1192/bjp.bp.1114.153767.

Hanlon C, Alem A, Medhin G, Shibre T, Ejigu DA, Negussie H, Dewey M, Wissow L, Prince M, Susser E, Lund C, Fekadu A (2016). Task sharing for the care of severe mental disorders in a low-income country (TaSCS): study protocol for a randomised, controlled, non-inferiority trial. Trials 17, 76. doi: 10.1186/s13063-13016-11191-x.
Lund C, Tomlinson T, De Silva M, Fekadu A, Shidhaye R, Jordans M, Petersen I, Bhana A, Kigozi F, Prince $M$, Thornicroft G, Hanlon C, Kakuma R, McDaid D, Saxena S, Chisholm D, Raja S, Kippen-Wood S, Honikman S, Fairall L, Patel V (2012). PRIME: a programme to reduce the treatment gap for mental disorders in five low- and middle-income countries. PLoS Medicine $\mathbf{9}$, e1001359.

Lund C, Waruguru M, Kingori J, Kippen-Wood S, Breuer E, Mannarath S, Raja S (2013). Outcomes of the mental health and development model in rural Kenya: a 2-year prospective cohort intervention study. International Health $\mathbf{5}$, $43-50$.

Mall S, Hailemariam M, Selamu M, Fekadu A, Lund C, Patel V, Petersen I, Hanlon C (2015). 'Restoring the person's life": a qualitative study to inform development of care for people with severe mental disorders in rural Ethiopia. Epidemiology and Psychiatric Sciences. doi: 10.1017/ S2045796015001006.

Mayston R, Alem A, Habtamu A, Shibre T, Fekadu A, Hanlon C (2015). Participatory planning of a primary care service for people with severe mental disorders in rural Ethiopia. Health Policy and Planning 31, 367-376. doi: 10.1093/heapol/czv1072.

Minoletti A, Galea S, Susser E (2012). Community mental health services in Latin America for people with severe mental disorders. Public Health Reviews 34, pii: http://www. publichealthreviews.eu/show/a/116.

Nadkarni A, Hanlon C, Bhatia U, Fuhr D, Ragoni C, de Azevedo Perocco SL, Fortes S, Shidhaye R, Kinyanda E, Rangaswamy T, Patel V (2015). The presentation and management of psychiatric emergencies in low and middle income countries: a systematic review. Lancet Psychiatry 2, 540-547.

Ng LC, Hanlon C, Yimer G, Henderson DC, Fekadu A (2015). Ethics in global health research: the need for balance. Lancet Global Health 3, e516-e517.

Ngo VK, Rubinstein A, Ganju V, Kanellis P, Loza N, Rabadan-Diehl C, Daar AS (2013). Grand challenges: integrating mental health care into the non-communicable disease agenda. PLoS Medicine 10, e1001443.

Nortje G, Oladeji B, Gureje O, Seedat S (2016). Effectiveness of traditional healers in treating mental disorders: a systematic review. Lancet Psychiatry 3, 154-170.

Padmanathan P, De Silva M (2013). The acceptability and feasibility of task-sharing for mental healthcare in low and middle income countries: a systematic review. Social Science and Medicine 97, 82-86.

Patel V (2016). Universal health coverage for schizophrenia: a global mental health priority. Schizophrenia Bulletin 42, 885890.

Petersen I, Marais D, Egbe CO, Alem A, Hanlon C, Shidhaye R, Abdulmalik J, Gureje O, Lund C, Chisholm D, Ahuja S, Upadhaya N, Jordans M, Mugisha J, Thornicroft G, Kigozi F (2016). Strengthening mental health system governance in six low-and middle-income countries in Africa and south Asia: challenges, needs and potential strategies. Health Policy and Planning (Under review). 
Purgato M, Adams C, Barbui C (2012). Schizophrenia trials conducted in African countries: a drop of evidence in the ocean of morbidity? International Journal of Mental Health Systems 6. doi: 10.1186/1752-4458-1186-1189.

Ran M-S, Yu-Hai Chen E, Conwell Y, Lai-Wan Chan C, Yip PSF, Xiang M-Z, Caine ED (2007). Mortality in people with schizophrenia in rural China: 10-year cohort study. British Journal of Psychiatry 190, 237-242.

Semrau M, Evans-Lacko S, Alem A, Ayuso-Mateos JL, Chisholm D, Gureje O, Hanlon C, Jordans M, Kigozi F, Lempp H, Lund C, Petersen I, Shidhaye R, Thornicroft G (2015). Strengthening mental health systems in low- and middle-income countries: the Emerald programme. $B M C$ Medicine 13, 1-9.

Shidhaye R, Shrivastava S, Murhar V, Samudre S, Ahuja S, Ramaswamy R, Patel V (2015). Development and piloting of a plan for integrating mental health in primary care in Sehore district, Madhya Pradesh, India. British Journal of Psychiatry 208, s13-20.

Strand KB, Chisholm D, Fekadu A, Johansson KA (2015). Scaling-up essential neuropsychiatric services in Ethiopia: a cost-effectiveness analysis. Health Policy and Planning 31, 504-513.

Thara R, Srinivasan TN (2000). How stigmatising is schizophrenia in India? International Journal of Psychiatry 46, 135-141.

Thornicroft G, Alem A, Antunes Dos Santos R, Barley E, Drake RE, Gregorio G, Hanlon C, Ito H, Latimer E, Law A, Mari J, McGeorge P, Padmavati R, Razzouk D, Semrau M, Setoya Y, Thara R, Wondimagegn D (2010). WPA guidelines on steps, obstacles and mistakes to avoid in the implementation of community mental health care. World Psychiatry 9, 67-77.

Trani J-F, Bakhshi P, Kuhlberg J, Narayanan SS, Venkataraman $\mathrm{H}$, Mishra NN, Groce NE, Jadhav S,
Deshpande S (2015). Mental illness, poverty and stigma in India: a case-control study. BMJ Open 5, e006355. doi: 006310.001136/bmjopen-002014-006355.

van Ginneken N, Tharyan P, Lewin S, Rao GN, Meera SM, Pian J, Chandrashekar S, Patel V (2013). Non-specialist health worker interventions for the care of mental, neurological and substance-abuse disorders in low- and middle-income countries. Cochrane Database of Systematic Reviews (11), CD009149.

Wagner EH, Austin BT, Davis C, Hindmarsh M, Schaefer J, Bonomi A (2001). Improving chronic illness care: translating evidence into action. Health Affairs 20, 64-78.

Wang PS, Angermeyer M, Borges G, Bruffaerts R, Chiu WT, de Girolamo G, Fayyad J, Gureje O, Haro JM, Huang Y, Kessler RC, Kovess V, Levinson D, Nakane Y, Brown MAO, Ormel JH, Posada-Villa J, Aguilar-Gaxiola S, Alonso J, Lee S, Heering S, Pennell B-E, Chatterji S, Ustun TB, for the WHO World Mental Health Survey Consortium (2007). Delay and failure in treatment seeking after first onset of mental health disorders in the World Health Organization's World Mental Health Survey Initiative. World Psychiatry 6, 177-185.

World Health Organization (2008). mhGAP Mental Health Gap Action Programme: Scaling up Care for Mental, Neurological and Substance use Disorders. WHO: Geneva.

World Health Organization (2010). mhGAP Intervention Guide for Mental, Neurological and Substance use Disorders in Non-specialized Health Settings: Mental Health Gap Action Programme (mhGAP). WHO: Geneva. http://www.who.int/mental_health/evidence/ mhGAP_intervention_guide/en/index.html.

Zhao S, Sampson S, Xia J, Jayaram MB (2015). Psychoeducation (brief) for people with serious mental illness. Cochrane Database of Systematic Reviews (4), CD010823. 